\title{
Elliptical Slot Microstrip Patch Antenna Design Based on a Dynamic Constrained Multiobjective Optimization Evolutionary Algorithm
}

\author{
Rangzhong Wu, School of Mechanical and Electronic Information, China University of Geosciences, Wuhan, China \\ Caie Hu, China University of Geosciences, Wuhan, China \\ Zhigao Zeng, School of Computer, Hunan University of Technology, China \\ Sanyou Zeng, School of Mechanical and Electronic Information, China University of Geosciences, Wuhan, China \\ (iD) https://orcid.org/0000-0002-0795-9092 \\ Jawdat S. Alkasassbeh, School of Mechanical and Electronic Information, China University of Geosciences, Wuhan, China
}

\begin{abstract}
Most evolutionary optimization algorithms have already been used for antenna design and shown promising results on improving the performance of the antenna. However, for many real-world antenna optimization problems, they are difficult to solve in that there are highly constrained and multimodal difficulties. These difficulties impede the development of antenna design. In this paper, an elliptical slot microstrip patch antenna design with these difficulties is modeled as a constrained optimization problem (COP). To address the problem, a dynamic constrained multiobjective optimization evolutionary algorithm (DCMOEA) is used. The experimental results show that the optimum antenna with satisfying the design requirement is obtained, and the radiation patch should be a whole ellipse instead of subtracting with two ellipses.
\end{abstract}

\section{KEYWORDS}

Antenna Design and Dynamic Constrained Multiobjective Optimization Problem, Constrained Optimization Problem, Evolutionary algorithms, Multimodal

\section{INTRODUCTION}

Evolutionary algorithms(EAs) are global, parallel, search and optimization methods, based on the principles of natural selection (Darwin, C. 2004) and population genetics (Fisher, R. A. 1958), and which are found a wide range of applications in various fields of science and engineering (Dasgupta, D., \& Michalewicz, Z. (Eds.). 2013, Fleming, P. J., \& Purshouse, R. C. 2002). EAs have been widely employed for solving antenna problems since the early 1990s (Altshuler, E. E., \& Linden, D. S. 1997, Linden, D. S., \& Altshuler, E. E. 1996), generally, antenna design problems are modeled as constrained optimization problems (COPs) (Hu, C., et al. 2019, Liu, B., et al. 2013, Liu, Z., Zeng, S., Jiang, Y., Li, C., \& Ouyang, N. 2013, Jia, L., et al. 2011, Wang, Y., \& Cai, Z. 2012,). Such as, Genetic Algorithm (GA) (Goldberg, D. E., \& Holland, J. H. 1988) is usually selected as an optimization tool 
for the antenna design in (Altshuler, E. E., \& Linden, D. S. 1997, Chou, H. T., \& Cheng, D. Y. 2016, Linden, D. S., \& Altshuler, E. E. 1996, Makki, B., Ide, A., Svensson, T., Eriksson, T., \& Alouini, M. S. 2016, Wen, Y. Q., Wang, B. Z., \& Ding, X. 2015). Differential Evolution (DE) (Storn, R., \& Price, K. 1997) is used frequently in the field of antenna design (Hu, C., et al. 2019, Cui, C. Y., Deb, A., Roy, J. S., \& Gupta, B. 2014, Ding, Y., Jiao, Y. C., Zhang, Jiao, Y. C., \& Zhang, L. 2017, L., \& Li, B. 2014). Particle Swarm Optimization (Eberhart, R., \& Kennedy, J. 1995) is used for antenna design (Jin, N., \& Rahmat-Samii, Y. 2007, Robinson, J., \& Rahmat-Samii, Y. 2004). Evolution Strategy (ES) (Rechenberg, I. 1994) is used usually in the antenna optimization (Chen, Y., Yang, S., \& Nie, Z. 2008, Choi, K., et al. 2015), and other evolutionary techniques.

Microstrip patch antennas with low profile, conformable to planar and nonplanar surfaces, easy realization and inexpensive to manufacture using modern printed circuit technology, have been widely researched in recent years (Colles, D., \& Arakaki, D. 2014, Dwivedi, S., Yadav, S. G., \& Singh, A. K. 2014, Radavaram, S., \& Pour, M. 2018, Pozar, D. M. 1992, Wang, H., Huang, X. B., Fang, D. G., \& Han, G. B. 2007), and pay an important role in developing the mobile radio, wireless communication, high-performance aircraft, and satellite engineering application. The patch shapes with ellipse that have a good demand and fame for their feed line flexibility and multiple frequency operation are usually considered in microstrip patch antennas design. On top of that, elliptical microstrip patch antennas that can give dual resonant frequency owing to two modes, even and odd mode (Agrawal, A., Vakula, D., \& Sarma, N. V. S. N. 2011, Long, S., Shen, L., Schaubert, D., \& Farrar, F. 1981). Therefore, there are many researchers have interested in elliptical patch microstrip antennas (Jung, H., \& Seo, C. 2002, Saxena, P., Ranjan, R., \& Gupta, K. 2016, Tasnim, N., Inum, R., Khatun, H., \& Khan, M. A. G. 2019).

A well-designed practical antenna should meet the requirements for the performance parameters of the antenna, for instance, Gain, VSWR, Axial ratio, Bandwidth etc. In extreme situations, the requirements are too high to solve the antenna problems. Besides, the antenna problems are a nonlinear problem. Traditional optimizers could not solve the problem well while EAs have potentiality in solving such complex problems. To address the problems, an efficient algorithm is indispensable for achieving the requirements needed. In generally, these problems are modeled as COPs. In extreme conditions, these COPs are highly COPs and not able to be solved by existing some constrained optimization methods. Such as, penalty function, it is hard to adjust the penalty coefficient $\lambda$, resulting in the feasible region is difficult to be obtained. The separation of objectives and constraints, it is easy to fall into a local optimum and the parameter need to be setted. At the present stage, there are few researches on solving the highly COPs. Besides, some the COPs are multimodal difficulty. In this paper, an existing work with great potential, that is, Dynamic Constrained Multiobjective Optimization Evolutionary Algorithm(DCMOEA) (Zeng, S., Jiao, R., Li, C., Li, X., \& Alkasassbeh, J. S. 2017) will be used for solving the antenna problems with highly constrained and multimodal difficulty.

In this paper, an elliptical slot microstrip patch antenna structure is a square substrate with a size of $30 \mathrm{~mm} * 30 \mathrm{~mm} * 1.6 \mathrm{~mm}$. The radiation patch on the front side, which is formed by subtracting two ellipses with the same axial ratio $R$. The coupling element in the back side, where consists of the difference between ground plate and an ellipse with an axial ratio $R$. The antenna design is modeled as a COP with highly constrained and multimodal difficulty and optimized by DCMOEA.

After a brief introduction in Section I, this paper is organized as follows: Section II introduces some related work. Section III gives the formation of antenna design as a COP. Then the antenna design problem is solved by DCMOEA in Section IV. Finally, Section V summarizes the conclusion of this paper.

\section{RELATED WORK}

In this section, we will review over the basic concept of the COP and introduce the existing DCMOEA. For antenna design, in general, the antenna problems often are modeled as a COP, which usually is a 
nonlinear problem. Furthermore, it is highly constrained and multimodal. For the complex antenna design problems, a number of traditional optimization methods are less than satisfactory while EAs have potentiality in solving them. To address such a practical problem, an existing DCMOEA has been proposed. The main purpose of the algorithm is to solve COP with highly constrained and multimodal difficulty, which based on the idea that a COP is converted into an equivalent dynamic constrained multiobjective optimization problem (DCMOP), including three objectives. Therefore, it is applied to this paper to solve the antenna design problem.

\section{The Basic Concepts of The COP}

\section{Definition 1. (Constrained Optimization Problem(COP))}

A general COP consists of an objective function, a set of $m$ constraints and $n$ decision variables. The objective and constraints are functions of the decision variables. Minimization optimization is considered in this paper. A COP can be mathematically defined as follows:

$$
\begin{aligned}
& \min y=f(\mathbf{x}) \\
& \text { st }: \mathbf{g}(\mathbf{x})=\left(g_{1}(\mathbf{x}), g_{2}(\mathbf{x}), \cdots, g_{m}(\mathbf{x})\right) \leq \mathbf{0} \\
& \text { where } \mathbf{x}=\left(x_{1}, x_{2}, \cdots, x_{n}\right) \in \mathbf{X} \\
& \mathbf{X}=\{\mathbf{x} \mid \mathbf{l} \leq \mathbf{x} \leq \mathbf{u}\} \\
& \mathbf{l}=\left(l_{1}, l_{2}, \cdots l_{n}\right), \mathbf{u}=\left(u_{1}, u_{2}, \cdots, u_{n}\right)
\end{aligned}
$$

where $f(\boldsymbol{x})$ is the objective function, $\boldsymbol{g}(\boldsymbol{x}) \leq 0$ is the constraint, $\mathbf{0}$ is the constrained boundary. $\boldsymbol{x}$ is the solution vector and $\boldsymbol{X}$ denotes the solution space, $\boldsymbol{l}$ and $\boldsymbol{u}$ are the lower and upper bounds of the solution space, respectively.

Definition 2. (Feasible solution and Feasible set)

A solution $\mathbf{x}=\left(x_{1}, x_{2}, \cdots, x_{n}\right) \in \mathbf{X}$ is feasible, if $\mathbf{g}(\mathbf{x}) \leq \mathbf{0}$, and vice versa. The Feasible set of a COP is defined as:

$S_{F}=(\mathbf{x}: \mathbf{x} \in \mathbf{X}, \mathbf{g}(\mathbf{x}) \leq \mathbf{0}\}$

Definition 3. (Constrained Violation)

Given a solution $\boldsymbol{x}$, the Constrained Violation of a constraint in Eq. (1) is usually defined as:

$$
G_{i}(\mathbf{x})=\max \left\{g_{i}(\mathbf{x}), 0\right\}, i=1,2, \cdots, m
$$

Definition 4. (Solution Violation)

Given a solution $\boldsymbol{x}$, the Constrained Violation of the solution is defined as: 
Algorithm 1. Comparison of $\boldsymbol{x}_{1}$ and $\boldsymbol{x}_{2}$

Case1 Both are feasible, then the one with smaller objective $f$ wins.

Case2 One is feasible and the other is infeasible, then the feasible one wins.

Case 3 Both are infeasible, then the one with smaller violation $\mathrm{CV}$ wins.

$c v(\mathbf{x})=\frac{1}{m} \sum_{i=1}^{m} \frac{G_{i}(\mathbf{x})}{\max _{\mathbf{x} \in P(0)}\left\{G_{i}(\mathbf{x})\right\}}$

where $P(0)$ is the initial population.

\section{Definition 5. (Niche-Count)}

Niche-count, is proposed by Goldberg and Richardson (Goldberg, D. E., \& Richardson, J.1987), aiming to prevent the population from trapping into a local optimum. Given $U=\left\{\boldsymbol{x}_{1}, \boldsymbol{x}_{2}, \cdots, \boldsymbol{x}_{L}\right\}$, the union of the parent and offspring population, and $\sigma$ is the niche radius. The niche-count $\boldsymbol{x} \in \mathrm{U}$ is defined as follows:

$$
n c=(\mathbf{x} \mid \mathbf{U}, \sigma)=\sum_{i=1, \mathbf{x}_{i} \neq \mathbf{x}}^{L} \operatorname{sh}\left(\mathbf{x}, \mathbf{x}_{i}\right)
$$

Where the sharing function between two solution $\boldsymbol{x}_{1}$ and $\boldsymbol{x}_{2}$ is denoted as:

$$
\operatorname{sh}\left(\boldsymbol{x}_{1}, \boldsymbol{x}_{2}\right)=\left\{\begin{array}{c}
1-\left(\frac{d\left(\boldsymbol{x}_{1}, \boldsymbol{x}_{2}\right)}{\sigma}\right), d\left(\boldsymbol{x}_{1}, \boldsymbol{x}_{2}\right)<\sigma \\
0, \text { otherwise }
\end{array}\right.
$$

where $d\left(\boldsymbol{x}_{1}, \boldsymbol{x}_{2}\right)$ is Euclidean distance between $\boldsymbol{x}_{1}$ and $\boldsymbol{x}_{2}$.

The order of two solutions is usually given as Algorithm 1, the comparison operator is to determine which one is better in comparison of two solutions (Dwivedi, S., Yadav, S. G., \& Singh, A. K. 2014).

Introduction of DCMOEA

DCMOEA demonstrates superior performance in handling COPs, in particular to the kind of problems with highly constrained and multimodal properties. The main contribution of the algorithm is that converting a COP into dynamic constrained multiobjective optimization problem (DCMOP) and to develop a general algorithm framework for solving DCMOPs by using multi-objective EAs (MOEAs). Due to the algorithm combines both multiobjective EAs technique and constraint handling mechanism. Therefore, there are lots of promising performances. The main three different properties in the algorithm as follows: 
1. A COP is converted into an equivalent DCMOP with three objectives, including an original objective, a constraint-violation objective and a niche- count objective.

2. A method of gradually reducing the constraint boundary in order to handle the constraint difficulty.

3. A method of gradually reducing the niche size in order to handle the multimodal difficulty.

There are three objectives for the equivalent DCMOP in the algorithm, the original objective, the constraint violation Eq. (3) and niche-count objective Eq. (4). Thus, a Eq. (1) COP will be converted into a DCMOP as follows:

$$
\begin{aligned}
& \min y=\left(f(\mathbf{x}), c v(\mathbf{x}), n c\left(\mathbf{x} \mid U, \sigma^{(s)}\right)\right) \\
& s t: \mathbf{g}(\mathbf{x})=\left(g_{1}(\mathbf{x}), g_{2}(\mathbf{x}), \cdots, g_{m}(\mathbf{x})\right) \leq \boldsymbol{\mu}^{(s)} \\
& \text { where } \mathbf{x}=\left(x_{1}, x_{2}, \cdots, x_{n}\right) \in \mathbf{X} \\
& \mathbf{X}=\{\mathbf{x} \mid \mathbf{l} \leq \mathbf{x} \leq \mathbf{u}\} \\
& \mathbf{l}=\left(l_{1}, l_{2}, \cdots l_{n}\right), \mathbf{u}=\left(u_{1}, u_{2}, \cdots, u_{n}\right)
\end{aligned}
$$

where $\mathrm{S}$ is a given number of environmental changes, and $\mathrm{S}$ is the environment state. $\boldsymbol{A}^{(s)}$ is the dynamic niche radius, $\boldsymbol{\mu}^{(s)}$ is the dynamic constraint boundary. $\boldsymbol{\sigma}^{(0)}>\boldsymbol{\sigma}^{(1)}>\cdots>\boldsymbol{\sigma}^{(S)}=\mathbf{0}$. $\boldsymbol{\mu}^{(s)}=\left(\varepsilon_{1}^{(s)}, \varepsilon_{1}^{(s)}, \cdots, \varepsilon_{m}{ }^{(s)}\right), \boldsymbol{\mu}^{(0)}>\boldsymbol{\mu}^{(1)}>\cdots \boldsymbol{\mu}^{(S)}=0, s=0,1, \cdots, S$. An environmental

change means that the reduction of the constraint boundary and the niche radius from state $s$ to state $s+1$.

Comparing the original COP Eq. (1) with DCMOP Eq. (6), at the final state $\mathrm{S}, \boldsymbol{\sigma}^{(S)}=\mathbf{0}, \boldsymbol{\mu}^{(S)}=0$, it is easy to find that $c v(\boldsymbol{x})=0$ and $n c(\boldsymbol{x} \mid U, 0)=0$. Thus, the DCMOP is equivalent to the COP, which is denoted as DCMOP $\cong \mathrm{COP}$.

Reducing gradually the constraint boundary from a large enough initial value to zero, will allow more solutions in the population are feasible as much as possible. The constraint handling mechanism is effective for handling the highly COP. The evolutionary process of the population over the mechanism is illustrated in Figure 1.

Also, reducing gradually the niche radius from an initial value to zero, allows the distribution of the population more diverse with the increase of niche radius $\sigma$. The exploration handling mechanism is effective for handling the multimodal property of the optimization problems.

In DCMOEA, the proposed $\mu$ constraint concept for the DCMOP as follows:

Definition 7. ( $\varepsilon$ feasible, $\varepsilon$ infeasible)

A solution $\mathbf{x}=\left(x_{1}, x_{2}, \cdots, x_{n}\right) \in \mathbf{X}$ is $\boldsymbol{\varepsilon}$ feasible, if $\mathbf{g}(\mathbf{x}) \leq \boldsymbol{\mu}$, and vice versa. The framework of DCMOEA for DCMOPs is shown as follows:

\section{Formulating antenna design as COP}

In this section, based on the design requirements considered, the design of the elliptical slot microstrip patch antenna is modeled as a COP. 
Figure 1. The evolutionary process of population over the constraint handling mechanism.

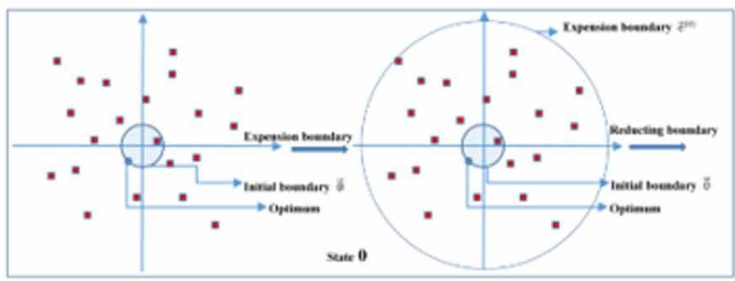

(a) State 0

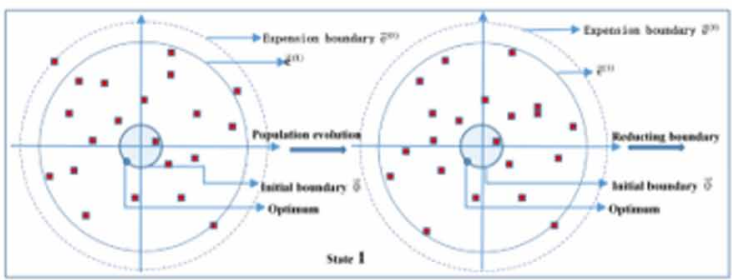

(b) State 1

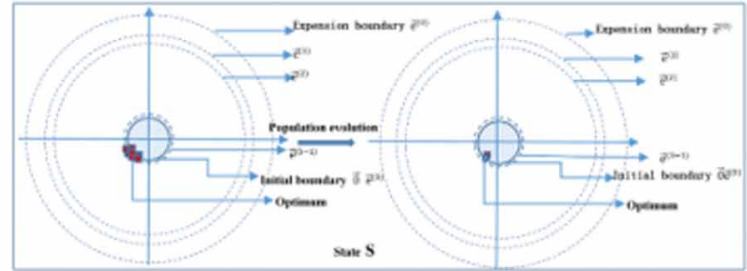

(c) State $S$

\section{Design Requirements of The Elliptical Slot Microstrip Patch Antenna}

Our purpose is to design an elliptical slot microstrip patch antenna with meeting the design requirement as shown in Table 1.

\section{Geometric Structure of The Elliptical Slot Microstrip Patch Antenna}

The geometric structure of the antenna with parameters is shown in Fig. 2.

The square flame retardant 4 (FR4) substrate has a side length of $30 \mathrm{~mm}$, the thickness of $1.6 \mathrm{~mm}$, the relative permittivity of 4.4, and the input resistance is $50 \Omega$. Considering the ellipse smoother than

\section{Algorithm 2 Algorithm Framework}

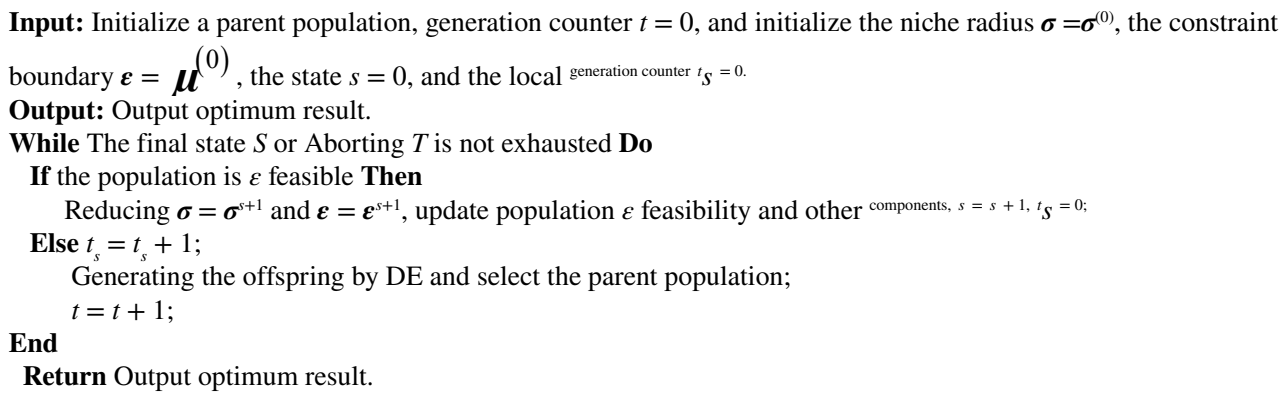


Table 1. Antenna design requirements

\begin{tabular}{|c|c|}
\hline Parameters & Requirements \\
\hline Frequency & The range from $2.35 \mathrm{GHz}$ to $2.55 \mathrm{GHz}$ \\
\hline Input impedance & $50 \Omega$ \\
\hline VSWR & $\leq 2$ \\
\hline Gain & $\geq 0 \mathrm{~dB}$ \\
\hline Pattern Range & $0 \circ \leq \theta \leq 180 \circ ; \varphi=0 \circ$ \\
\hline Size & $30 \mathrm{~mm} \times 30 \mathrm{~mm} \times 1.6 \mathrm{~mm}$ \\
\hline
\end{tabular}

the rectangle, the radiation patch on the front side, which is formed by subtracting two ellipses with the same axial ratio $\mathrm{R}$. The coupling element on the back side, that is the difference between the ground plate and an ellipse with an axial ratio $\mathrm{R}$. The feed point is located at bottom the side the antenna.

\section{Solution Vector and Solution Space}

Both the location and the size of the ellipses and the microstrip feed line are changeable, as shown in Fig. 2, where, $d 1$ and $d 2$ are the long axis of the frontal two ellipses with axial ratio $R$ respectively, and $(0, y 2)$ is the center of the ellipses. $d 3$ is the long axis of the back ellipse with axial ratio $R$, and $(0, y 1)$ is the center of the ellipse. $f w$ is the width of the feedline.

A coordinate system is constructed in the patch antenna with the origin at the center of the patch antenna, as seen in Fig. 2. The geometric structure of the antenna is determined by seven variables. Six variables $d 1, d 2, d 3, R, y 1$ and $y 2$ are to determine the structure of the ellipses, and the variable $f w$ is to determine the size of the microstrip feedline.

Figure 2. Geometric structure of the elliptical slot microstrip patch antenna in plane

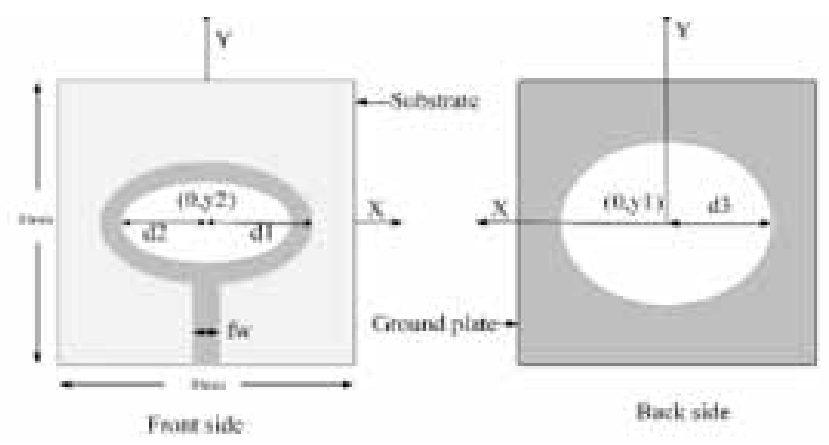

All those seven variables $\boldsymbol{x}=(d 1, d 2, d 3, R, f w, y 1, y 2)$ represent of the decision vectors. And the solution space is the ranges of all the seven variables, see Eq (7).

$\mathbf{X}=\{\mathbf{x} \mid \mathbf{l} \leq \mathbf{x} \leq \mathbf{u}\}$

$\mathbf{x}=(d 1, d 2, d 3, R, f w, y 1 . y 2)$

$\mathbf{l}=(10,0,0,0.6,1,-2.8,-2.8)$

$\mathbf{u}=(14,10,14,0.8,3,2.8,2.8)$ 


\section{Objective and Constraints}

The objective and constraints are modeled according to the design requirements in Table 3.1. What's more, the robustness of the antenna optimized also is taken into account in this paper. In practice, it is essential to concern about the robustness of antenna, specially for real engineering. The gain is sampled in $5^{\circ}$ increments over the region $0^{\circ} \leq \theta \leq 180^{\circ}$ and $\varphi=0^{\circ}$, and the frequencies are sampled in $5 \mathrm{MHz}$ increments over the band from $2.35 \mathrm{GHz}$ to $2.55 \mathrm{GHz}$.

In this paper, the objective function $f(\boldsymbol{x})$ is defined based on the robustness property is taken into account, which is based on our previous work (Hu, C., et al. 2019), see Eq (8).

$$
f(\mathbf{x})=\sum_{\theta} \sum_{\varphi}(\operatorname{Gvariance}(\theta, \varphi)+A \operatorname{Rvariance}(\theta, \varphi)+\operatorname{VSWRvariance})
$$

where $(\theta, \varphi)$ represents one direction in spherical coordinates, $\theta$ is the elevation and $\varphi$ is the azimuth angle with $0^{\circ}$. Gvariance $(\theta, \varphi)$, ARvariance $(\theta, \varphi)$ and $V S W R$ variance are the variances of gain, axial ratio and VSWR respectively over the frequency band.

The details of the objective function are shown as follows:

$$
\begin{aligned}
& \operatorname{Gvariance}(\theta, \varphi)=\frac{1}{\operatorname{len}(f r)} \sum_{f r}\left(\operatorname{Gain}(\theta, \varphi, f r)-\operatorname{Mean}_{(\theta, \varphi)}\right)^{2} \\
& \left.\operatorname{Mean}_{(\theta, \varphi)}\right)=\sum_{f r} \frac{\operatorname{Gain}(\theta, \varphi, f r)}{\operatorname{len}(f r)} \\
& \operatorname{ARvariance}_{(\theta, \varphi)}=\frac{1}{\operatorname{len}(f r)} \sum_{f r}\left(\operatorname{Axial}_{(\theta, \varphi, f r)}-\operatorname{MeanAR}_{(\theta, \varphi)}\right)^{2} \\
& \operatorname{MeanAR}_{(\theta, \varphi)}=\sum_{f r} \frac{\operatorname{Axial} l_{(\theta, \varphi, f r)}}{\operatorname{len}(f r)} \\
& \operatorname{VSWRvariance}=\frac{1}{\operatorname{len}(f r)} \sum_{f r}\left(\operatorname{VSWR} R_{f r}-\operatorname{MeanVSWR}_{f r}\right)^{2} \\
& \text { MeanVSWRfr }=\sum_{f r} \frac{\operatorname{VSWRfr}}{\operatorname{len}(f r)}
\end{aligned}
$$

where $f_{r}$ stands for a single frequency point, and $l e n_{f_{r}}$ is the number of points over the frequency band. Therefore, $\operatorname{Gain}(\theta, \varphi, f r), \operatorname{Axial}_{(\theta, \varphi, f r)}$ and $V S W R_{f r}$ represent the gain, axial ratio and VSWR respectively in direction $(\theta, \varphi)$ and at frequency point $f_{r}$.

The constraint on the gain of the antenna design problem is as shown in Eq (10).

$$
\operatorname{gain}_{\left(\theta, 0^{\circ}, f r\right)}(\mathbf{x})=-\operatorname{Gain}_{\left(\theta, 0^{\circ}, f r\right)} \leq 0
$$

The constraint on VSWR is defined over the frequency band, see Eq (11).

$$
g V S W R_{f r}(\mathbf{x})=V S W R_{f r}-2 \leq 0
$$


From the above, the antenna design is formed as a COP as follows:

$$
\begin{aligned}
& \min f(\mathbf{x})=V S W R \text { variance }+\sum_{\theta=0^{\circ}}^{\theta=180^{\circ}}\left(\text { Gvariance }\left(\theta, 0^{\circ}\right)+\text { ARvariance }\left(\theta, 0^{\circ}\right)\right) \\
& g V S W R_{f r}(\mathbf{x})=V S W R_{f r}-2 \leq 0 \\
& \mathbf{x}=(d 1, d 2, d 3, R, f w, y 1 . y 2) \\
& \mathbf{l}=(10,0,0,0.6,1,-2.8,-2.8) \\
& \mathbf{u}=(14,10,14,0.8,3,2.8,2.8)
\end{aligned}
$$

In above COP, the objective function $f(\boldsymbol{x})$ is assumed to be minimized. As we all know, the smaller the variances are, the more robust the antenna is.

\section{SOLVING ANTENNA DESIGN BY USING DCMOEA}

In this section, the DCMOEA is used as an optimization tool to search the optimum structure of the elliptical slot microstrip antenna. The optimum antenna will be obtained over performing the DCMOEA, and the results are discussed.

\section{Setting of DCMOEA}

The parameters for the DCMOEA in solving the antenna design are listed as follows

1. Evolutionary generations $T=500$.

2. Population size $N P=30$.

3. The settings of the DE operator: crossover probability $C R=0.9$, mutation probability $F=0.5$.

4. The two constant parameters in DCMOEA: precision requirement $\delta=1.0 e-8$, control parameter of the decreasing $c p=2$.

5. The number of environmental changes: $S=200$.

\section{RESULTS AND DISCUSSION}

The electromagnetic simulation software Ansoft HFSS 14.0 is employed to evaluate the antenna. The experiments were run on a PC with 32-bit Intel(R)Core(TM) processor 2.33GHz, Quad-Core, Memory 4G. After the DCMOEA is performed, the optimum geometric structure of the antenna is obtained, as shown in Fig. 3, and subject to the variables value of the evolved antenna are $(d 1=13.7$, $d 2=1.7, d 3=14, R=0.7448, f w=2.2, y 1=0.3, y 2=1.9)$. We have fabricated this antenna by $3 \mathrm{D}$ metal printing. A photo of a prototype is shown in Figure 4, the left side of the figure is the back of the evolved antenna, the right in front.

Performances of Evolved Antenna VSWR of the evolved antenna by DCMOEA is less than 2 over the all frequency from $2.35 \mathrm{MHz}$ to $2.55 \mathrm{MHz}$ as shown in Fig. 5. When assuming that the antenna can work in a real environment when its VSWR is less than 2. Based on this assumption, the bandwidth of the evolved antenna covers the whole frequency range. This means that the bandwidth of the evolved antenna is wide with $200 \mathrm{MHz}$ that satisfies the requirements table. Thus, an antenna with robust performance over the whole frequency range is attained by the algorithm.

At the same time, the gain is greater than 0 over the range $0^{\circ} \leq \theta \leq 180^{\circ}$ and $\varphi=0^{\circ}$ is shown in Figure 6. It indicates that the antenna evolved satisfies fully the design requirements. The threedimensional radiation pattern over the range $0^{\circ} \leq \theta \leq 180^{\circ}$ and $\varphi=0^{\circ}$ in center frequency $2.45 \mathrm{GHz}$ is shown in Figure 7. The antenna design problem is well solved by the DCMOEA, and attaining 
International Journal of Cognitive Informatics and Natural Intelligence

Volume 15 • Issue 4 • October-December 2021

Figure 3. The optimum geometric structure of the evolved antenna

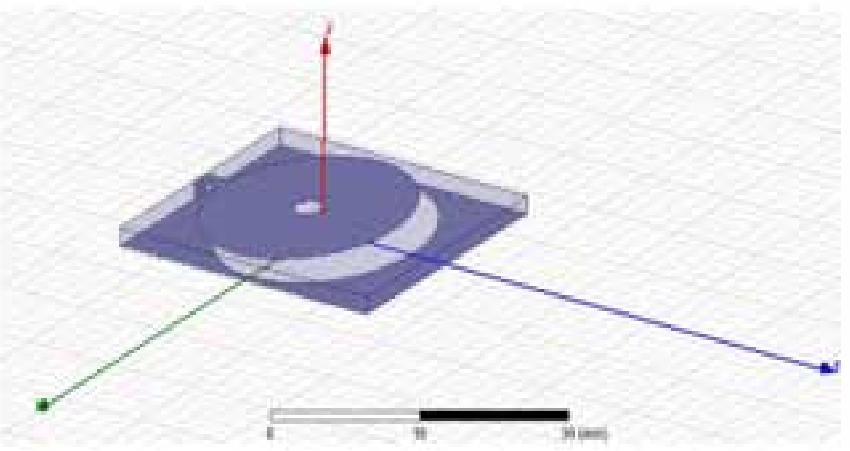

Figure 4. The prototype of the evolved antenna by 3D printing

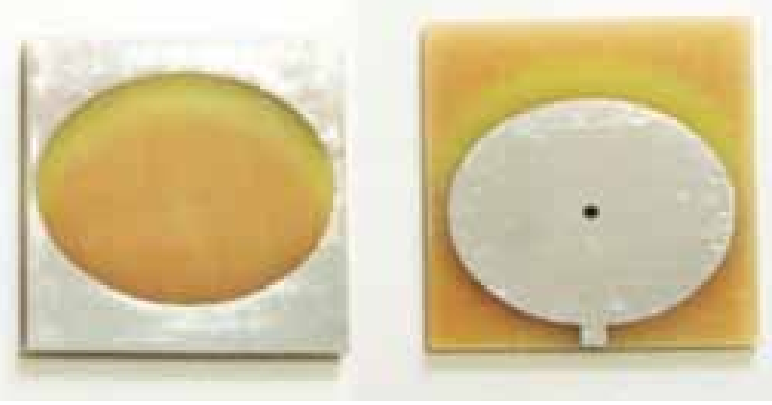

Figure 5. VSWR of the evolved antenna

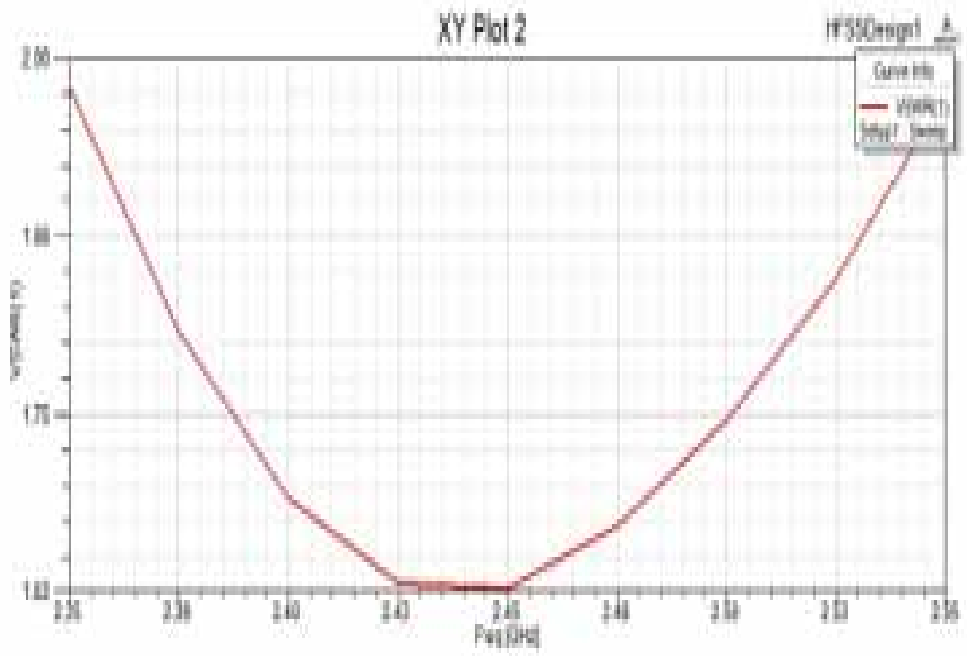


Figure 6. Gain of the evolved antenna

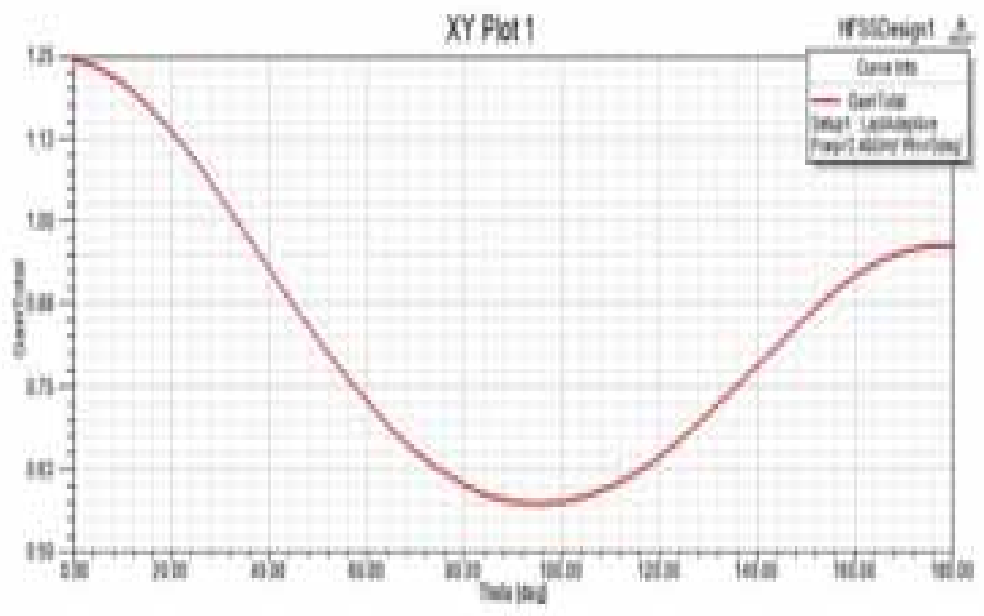

Figure 7. Radiation patterns of the evolved antenna
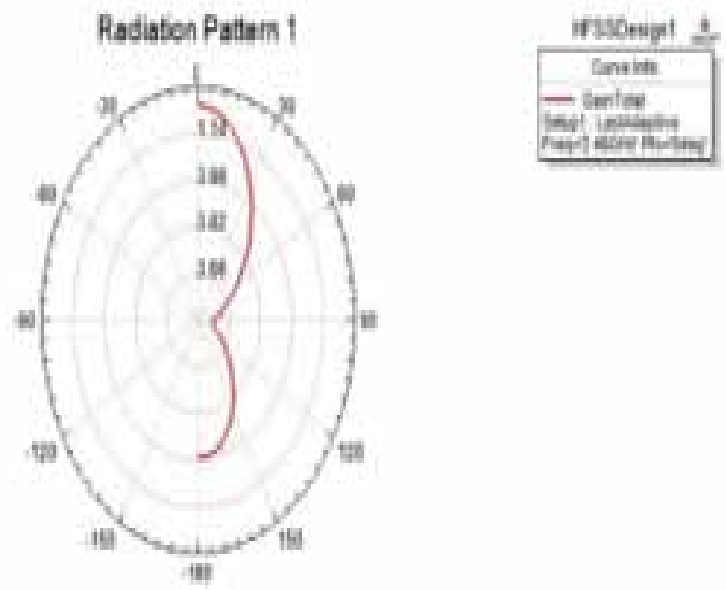

Figure 8. Application of the evolved antenna 
promising performance. Besides, it means that the DCMOEA has the potentiality to solve the complex antenna design problems.

It is worth noting that analyzing the structure and results of the evolved antenna via using DCMOEA, we find that the radiation patch should be a whole ellipse instead of subtracting with two ellipses since $\mathrm{d} 2=1.7$ is largely less than $1 / 10$ wavelength. In surface antennas, as the proposed antenna in this paper, effective aperture is an important indicators for an antenna to measure the ability to receive radio wave. Based on the surface antennas theory for the effective aperture, a large effective aperture over the entire scan range is usually needed. In other words, the larger the effective aperture is, the better the performance of the antenna is.

In our experiment, based on the results of the evolved antenna, the radiation patch obtained by optimizing is a whole ellipse instead of subtracting with two ellipses, thus, the performance of the antenna is good. It demonstrates that our experiment conforms to the theory of the surface antennas, as well as can obtain remarkable performance. Therefore, we draw a conclusion that the radiation patch should be complete rather than partial, which provides a valuable reference for follow-up study with antenna design in real engineering and scientific research.

\section{Application of the evolved antenna}

In Figure 8, the evolved antenna is mounted to the router in the lab of our evolutionary antenna optimization group at China University of Geosciences, which is used to test whether the evolved antenna can work. The results shown that the antenna works well.

\section{CONCLUSION AND FUTURE RESEARCH}

This paper shows that evolving an elliptical slot microstrip patch antenna by using DCMOEA. It has been shown that

1. Modeling the design problem of the elliptical slot microstrip patch antenna as a COP, and solving the problem by DCMOEA

2. At the same time, the evolved antenna by the DCMOEA has obtained remarkable performance.

3. Otherwise, the evolved antenna is mounted to the router in the lab of our evolutionary antenna optimization group at China University of Geosciences, and it showed works well.

4. Last but not least, it is noteworthy that, we find that the radiation patch should be a whole ellipse instead of subtracting with two ellipses. It conforms to the theory of the surface antennas. Therefore, we draw a conclusion that the radiation patch should be complete rather than partial in the surface antennas design, which provides a valuable reference for follow-up study with antenna design in real engineering and scientific research.

In the future research, we will focus on applying the expensive optimization algorithm for the elliptical slot microstrip patch antenna to reduce the computationally expensive.

\section{ACKNOWLEDGMENT}

This work was supported by Major Project for New Generation of AI Grant No.2018AAA0100400, the National Natural Science Foundation of China and other foundations (No.s: 61673355,61271140, 61203306, 2012001202, 60871021), and high-performance computing platform of China University of Geosciences. 


\section{REFERENCES}

Agrawal, A., Vakula, D., \& Sarma, N. V. S. N. (2011, September). Design of elliptical microstrip patch antenna using ANN. PIERS Proceedings.

Altshuler, E. E., \& Linden, D. S. (1997). Wire-antenna designs using genetic algorithms. IEEE Antennas \& Propagation Magazine, 39(2), 33-43. doi:10.1109/74.584498

Chen, Y., Yang, S., \& Nie, Z. (2008). The application of a modified differential evolution strategy to some array pattern synthesis problems. IEEE Transactions on Antennas and Propagation, 56(7), 1919-1927. doi:10.1109/ TAP.2008.924713

Choi, K., Jang, D. H., Kang, S. I., Lee, J. H., Chung, T. K., \& Kim, H. S. (2015). Hybrid algorithm combing genetic algorithm with evolution strategy for antenna design. IEEE Transactions on Magnetics, 52(3), 1-4. doi:10.1109/TMAG.2015.2486043

Chou, H. T., \& Cheng, D. Y. (2016). Beam-pattern calibration in a realistic system of phased-array antennas via the implementation of a genetic algorithm with a measurement system. IEEE Transactions on Antennas and Propagation, 65(2), 593-601. doi:10.1109/TAP.2016.2635630

Colles, D., \& Arakaki, D. (2014, July). Multi-technique broadband microstrip patch antenna design. In 2014 IEEE Antennas and Propagation Society International Symposium (APSURSI) (pp. 1879-1880). IEEE. doi:10.1109/ APS.2014.6905266

Cui, C. Y., Jiao, Y. C., \& Zhang, L. (2017). Synthesis of some low sidelobe linear arrays using hybrid differential evolution algorithm integrated with convex programming. IEEE Antennas and Wireless Propagation Letters, 16, 2444-2448. doi:10.1109/LAWP.2017.2723568

Darwin, C. (2004). On the origin of species (Vol. 1859). Routledge.

Dasgupta, D., \& Michalewicz, Z. (Eds.). (2013). Evolutionary algorithms in engineering applications. Springer Science \& Business Media.

Deb, A., Roy, J. S., \& Gupta, B. (2014). Performance comparison of differential evolution, particle swarm optimization and genetic algorithm in the design of circularly polarized microstrip antennas. IEEE Transactions on Antennas and Propagation, 62(8), 3920-3928. doi:10.1109/TAP.2014.2322880

Deb, K. (2000). An efficient constraint handling method for genetic algorithms. Computer Methods in Applied Mechanics and Engineering, 186(2-4), 311-338. doi:10.1016/S0045-7825(99)00389-8

Ding, Y., Jiao, Y. C., Zhang, L., \& Li, B. (2014). Solving port selection problem in multiple beam antenna satellite communication system by using differential evolution algorithm. IEEE Transactions on Antennas and Propagation, 62(10), 5357-5361. doi:10.1109/TAP.2014.2341293

Dwivedi, S., Yadav, S. G., \& Singh, A. K. (2014). Annular ring embedded L-slot rectangular microstrip patch antenna. In Proceedings of the 2014 IEEE Students' Technology Symposium (pp. 372-375). IEEE.

Eberhart, R., \& Kennedy, J. (1995, November). Particle swarm optimization. In Proceedings of the IEEE international conference on neural networks (Vol. 4, pp. 1942-1948). IEEE.

Fisher, R. A. (1958). The genetical theory of natural selection. Рипол Классик.

Fleming, P. J., \& Purshouse, R. C. (2002). Evolutionary algorithms in control systems engineering: A survey. Control Engineering Practice, 10(11), 1223-1241. doi:10.1016/S0967-0661(02)00081-3

Goldberg, D. E., \& Holland, J. H. (1988). Genetic algorithms and machine learning. Academic Press.

Goldberg, D. E., \& Richardson, J. (1987, July). Genetic algorithms with sharing for multimodal function optimization. In Genetic algorithms and their applications: Proceedings of the Second International Conference on Genetic Algorithms (pp. 41-49). Hillsdale, NJ: Lawrence Erlbaum.

Hu, C., Zeng, S., Jiang, Y., Sun, J., Sun, Y., \& Gao, S. (2019). A Robust Technique Without Additional Computational Cost in Evolutionary Antenna Optimization. IEEE Transactions on Antennas and Propagation, 67(4), 2252-2259. doi:10.1109/TAP.2019.2891661 
Jia, L., Zeng, S., Zhou, D., Zhou, A., Li, Z., \& Jing, H. (2011, June). Dynamic multi-objective differential evolution for solving constrained optimization problem. In 2011 IEEE Congress of Evolutionary Computation (CEC) (pp. 2649-2654). IEEE.

Jin, N., \& Rahmat-Samii, Y. (2007). Advances in particle swarm optimization for antenna designs: Real-number, binary, single-objective and multiobjective implementations. IEEE Transactions on Antennas and Propagation, 55(3), 556-567.

Jung, H., \& Seo, C. (2002). Analysis of elliptical microstrip patch antenna considering attachment mode. IEEE Transactions on Antennas and Propagation, 50(6), 888-890.

Linden, D. S., \& Altshuler, E. E. (1996). Automating wire antenna design using genetic algorithms. Microwave Journal, 39(3), 74-81.

Liu, B., Aliakbarian, H., Ma, Z., Vandenbosch, G. A., Gielen, G., \& Excell, P. (2013). An efficient method for antenna design optimization based on evolutionary computation and machine learning techniques. IEEE Transactions on Antennas and Propagation, 62(1), 7-18. doi:10.1109/TAP.2013.2283605

Liu, Z., Zeng, S., Jiang, Y., Li, C., \& Ouyang, N. (2013, April). Evolutionary design of a wide-band twisted dipole antenna for X-band application. In 2013 IEEE International Conference on Evolvable Systems (ICES) (pp. 9-12). IEEE. doi:10.1109/ICES.2013.6613276

Long, S., Shen, L., Schaubert, D., \& Farrar, F. (1981). An experimental study of the circular-polarized elliptical printed-circuit antenna. IEEE Transactions on Antennas and Propagation, 29(1), 95-99. doi:10.1109/ TAP.1981.1142549

Makki, B., Ide, A., Svensson, T., Eriksson, T., \& Alouini, M. S. (2016). A genetic algorithm-based antenna selection approach for large-but-finite MIMO networks. IEEE Transactions on Vehicular Technology, 66(7), 6591-6595.

Pozar, D. M. (1992). Microstrip antennas. Proceedings of the IEEE, 80(1), 79-91.

Radavaram, S., \& Pour, M. (2018). Wideband radiation reconfigurable microstrip patch antenna loaded with two inverted U-slots. IEEE Transactions on Antennas and Propagation, 67(3), 1501-1508.

Rechenberg, I. (1994). Evolutionsstrategie: Optimierung technischer Systeme nach Prinzipien der biologischen Evolution. frommann-holzbog, Stuttgart, 1973. Step-Size Adaptation Based on Non-Local Use of Selection Information. In Parallel Problem Solving from Nature (PPSN3). Academic Press.

Robinson, J., \& Rahmat-Samii, Y. (2004). Particle swarm optimization in electromagnetics. IEEE Transactions on Antennas and Propagation, 52(2), 397-407.

Saxena, P., Ranjan, R., \& Gupta, K. (2016, February). Design and analysis of frequency agile elliptical patch microstrip antenna. In 2016 International Conference on Innovation and Challenges in Cyber Security (ICICCSINBUSH) (pp. 289-293). IEEE.

Storn, R., \& Price, K. (1997). Differential evolution-a simple and efficient heuristic for global optimization over continuous spaces. Journal of Global Optimization, 11(4), 341-359.

Tasnim, N., Inum, R., Khatun, H., \& Khan, M. A. G. (2019, January). Comparative Study on Circular and Elliptical Microstrip Patch Antenna Arrays with Microstrip Line and Coaxial Probe Feeding for X-band. In 2019 International Conference on Robotics, Electrical and Signal Processing Techniques (ICREST) (pp. 74-78). IEEE.

Wang, H., Huang, X. B., Fang, D. G., \& Han, G. B. (2007). A microstrip antenna array formed by microstrip line fed tooth-like-slot patches. IEEE Transactions on Antennas and Propagation, 55(4), 1210-1214.

Wang, Y., \& Cai, Z. (2012). Combining multiobjective optimization with differential evolution to solve constrained optimization problems. IEEE Transactions on Evolutionary Computation, 16(1), 117-134.

Weile, D. S., \& Michielssen, E. (1997). Genetic algorithm optimization applied to electromagnetics: A review. IEEE Transactions on Antennas and Propagation, 45(3), 343-353.

Wen, Y. Q., Wang, B. Z., \& Ding, X. (2015). A wide-angle scanning and low sidelobe level microstrip phased array based on genetic algorithm optimization. IEEE Transactions on Antennas and Propagation, 64(2), 805-810. 
Zeng, S., Jiao, R., Li, C., Li, X., \& Alkasassbeh, J. S. (2017). A general framework of dynamic constrained multiobjective evolutionary algorithms for constrained optimization. IEEE Transactions on Cybernetics, 47(9), 2678-2688.

Rangzhong Wu is a lecturer at School of Mechanical and Electronic Information, China University of Geosciences.

Caie Hu is currently pursuing the Ph.D degree in in control science and engineering from China University of Geoscience(Wuhan), China. Her current research interests include data-driven surrogate-assisted evolutionary optimization with machine learning for solving problems with constraints, dynamic environments, and expensive costs, especially the antenna design problems.

Zhigao Zeng is teacher at School of Computer, Hunan University of Technology.

Sanyou Zeng is a professor at School of Mechanical and Electronic Information, China University of Geosciences. His current research interests include evolutionary computation with machine learning for solving problems with constraints, multi-objective, dynamic environments, and expensive costs, especially the antenna design problems.

Jawdat S. Alkasassbeh is currently pursuing the Ph.D degree in in control science and engineering from China University of Geoscience(Wuhan),China. 